\title{
Cannulation strategies, circulation management and neuroprotection for type A intramural hematoma: tips and tricks
}

\author{
Bradley G. Leshnower \\ Division of Cardiothoracic Surgery, Department of Surgery, Emory University School of Medicine, Atlanta, GA, USA \\ Correspondence to: Bradley G. Leshnower. Associate Professor of Surgery, Division of Cardiothoracic Surgery, Emory University School of Medicine, \\ Atlanta, GA, USA. Email: bleshno@emory.edu.
}

\begin{abstract}
The circulation management strategies and operative techniques that are essential for the successful surgical repair of acute type A intramural hematoma (ATAIMH) are similar to those utilized for acute type A aortic dissection. This article reviews the neuroprotection strategies and arterial cannulation sites used in the treatment of patients with ATAIMH.
\end{abstract}

Keywords: Aortic dissection; arterial cannulation; intramural hematoma (IMH)

Submitted May 04, 2019. Accepted for publication Jul 25, 2019.

doi: $10.21037 /$ acs.2019.08.08

View this article at: http://dx.doi.org/10.21037/acs.2019.08.08

\section{Introduction}

Acute type A intramural hematoma (ATAIMH) has a mortality rate similar to acute type A aortic dissection, and urgent surgery remains the recommended therapy $(1,2)$. There is a $33 \%$ mortality rate associated with medical management of ATAIMH, compared to a $12 \%$ mortality for ATAIMH patients who undergo ascending aortic replacement $(3,4)$. Excellent neurologic outcomes and low mortality can be achieved with well-planned strategies for arterial cannulation and cerebral protection when combined with meticulous surgical technique. This article discusses the various options for arterial cannulation and the different methods of cerebral protection that can be utilized during the surgical repair of ATAIMH.

\section{Assessment of preoperative imaging}

As is the case with acute type A dissection, a detailed analysis of the preoperative computed tomography (CT) scan is vital to achieving intraoperative success. It should be noted that ATAIMH can be diagnosed on a non-contrast CT scan, radiographic signs indicative of rupture include: an irregular aortic contour, a blush of contrast into the IMH or outside the adventitia, or a pericardial effusion. Aortic diameters should be assessed at the root, and in the proximal, mid- and distal arch. Aortic root replacement and total arch replacement should be strongly considered if the sinus of Valsalva or mid-arch diameter is $\geq 4.5 \mathrm{~cm} \mathrm{(2).}$ The mid-arch should also be measured for graft sizing. A patent false lumen in the descending aorta in the setting of ATAIMH is suggestive of a type B aortic dissection with retrograde IMH in the ascending aorta. In this situation, an extended arch procedure with or without concomitant antegrade deployment of a thoracic aortic stent graft into the true lumen of the descending aorta is recommended to excise or cover the primary intimal tear. Finally, the great vessels should be carefully assessed for their anatomy and the presence of IMH in these branch vessels, as this can impact cannulation strategy. Intraoperative transesophageal echocardiogram should be used to assess: (I) aortic valve function; (II) the diameters of the sinus of Valsalva and sinotubular junction; (III) the presence of a pericardial effusion; and (IV) evidence of cardiac tamponade.

\section{Arterial cannulation}

Preoperative factors that may influence the site of arterial cannulation in the treatment of ATAIMH include radiographic signs and clinical symptoms of rupture (e.g., hemopericardium, tamponade), previous sternotomy and specific anatomic features of the IMH. Direct 


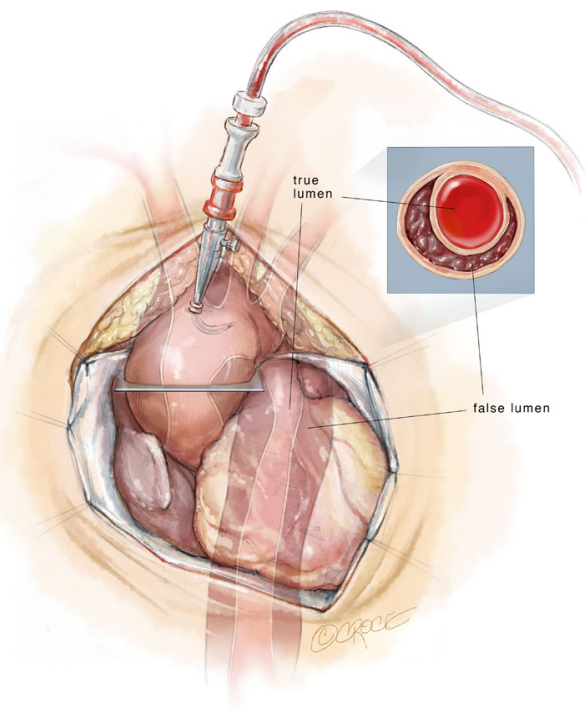

Figure 1 Ascending aortic cannulation in acute type A intra-mural hematoma. A cross section of the aorta depicts a thrombosed false lumen.

cannulation of the ascending aorta is the most expedient option for patients who require emergent initiation of cardiopulmonary bypass due to hemodynamic instability (Figure 1). If the IMH is $>5 \mathrm{~mm}$ and has circumferential involvement of the aorta, cannulation over a wire using the Seldinger technique is recommended as this may reduce the risk of introducing hematoma into the true lumen of the aorta during cannulation. Right axillary artery cannulation is an excellent option which has been shown to be associated with lower stroke rates in patients undergoing circulatory arrest for ascending aortic and arch replacement (5). At Emory, our preferred method of arterial cannulation for circulatory arrest cases is to anastomose an $8 \mathrm{~mm}$ Dacron graft sewn in an end-to-side fashion to the right axillary artery. This technique eliminates the risk of right arm malperfusion as opposed to direct cannulation (Figure 2). The benefits of right axillary artery cannulation include: (I) the ability to provide antegrade cerebral perfusion (ACP) with occlusion of the innominate artery during the period of circulatory arrest; (II) rapid initiation of cardiopulmonary bypass in the event of significant bleeding upon sternotomy in the reoperative or rupture setting; and (III) avoidance of cannulating the acutely disrupted, fragile ascending aorta in ATAIMH which may result in significant bleeding.

Frequently in ATAIMH, the innominate artery is spared from the disease process and can serve as another
A

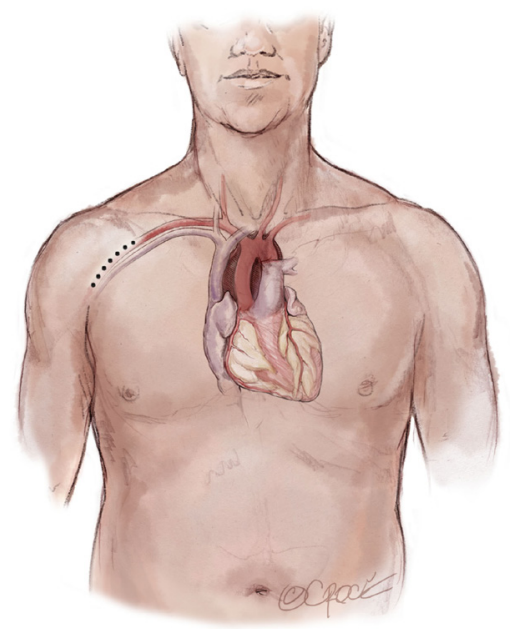

B

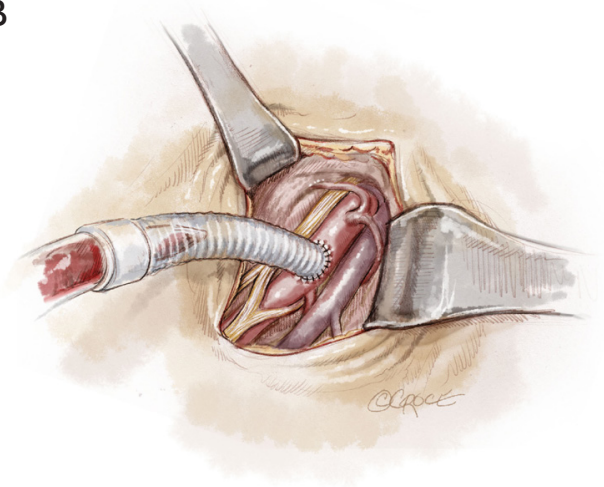

Figure 2 Right axillary artery cannulation. A $5 \mathrm{~cm}$ incision is made in the deltopectoral groove and the right axillary artery is isolated. An $8 \mathrm{~mm}$ Dacron graft is sewn end-to-side on to the right axillary artery and cannulated for arterial inflow for cardiopulmonary bypass.

cannulation site that facilitates the delivery of ACP during the period of circulatory arrest without an additional incision. Following sternotomy, the innominate artery is isolated cephalad to the innominate vein prior to opening the pericardium. As the vessel is of large caliber, a partial occlusion clamp can be utilized to isolate a segment of the artery while still maintaining perfusion to the right carotid and right subclavian arteries. Our preferred method is to anastomose an $8 \mathrm{~mm}$ Dacron graft sewn end-to-side to the innominate artery (Figure 3). In the reoperative setting, in which the right axillary artery has already been used, the right or left common carotid artery (typically spared in ATAIMH) is another easily accessible option. A $5 \mathrm{~cm}$ incision is made on the anterior aspect of the sternocleidomastoid, one fingerbreadth above the 
clavicle, and the common carotid artery is isolated. An $8 \mathrm{~mm}$ Dacron graft is sewn end-to-side to the common carotid and cannulated to provide both arterial inflow for cardiopulmonary bypass and ACP at the time of circulatory arrest (Figure 4). Finally, the femoral artery, which is

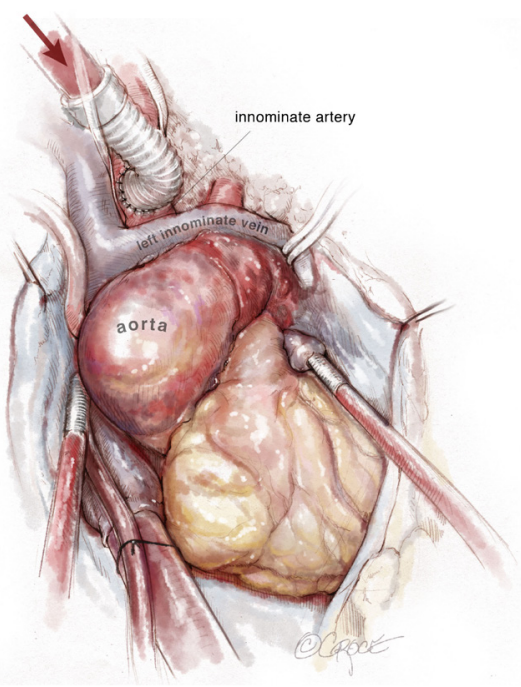

Figure 3 Innominate artery cannulation. Following sternotomy, the innominate artery is isolated with a partial occlusion clamp. An $8 \mathrm{~mm}$ Dacron graft is sewn end-to-side on to the innominate artery and cannulated for arterial inflow for cardiopulmonary bypass. typically spared in ATAIMH, remains an option for open or percutaneous femoral artery cannulation (Figure 5).

\section{Neuroprotection strategies}

Although the majority of patients with ATAIMH require ascending aortic replacement alone, it is highly recommended to perform the distal anastomosis with an open technique (no aortic cross-clamp) under circulatory arrest. Repair of the acutely disrupted ascending aorta under a cross clamp (without circulatory arrest) has been associated with a higher risk of stroke and reoperation on the ascending aorta (6-8). It is also recommended to avoid placing a cross-clamp on the ascending aorta prior to the circulatory arrest period, as we have demonstrated a 2.3fold higher incidence of stroke with aortic cross-clamping in acute type A aortic dissection, even with an open distal anastomotic technique (9). Given the increased risk of adverse neurologic events in the repair of all acute aortic syndromes using circulatory arrest, cerebral protection strategies must be employed to mitigate the risk. We recommend using intraoperative neuromonitoring, consisting of a continuous electroencephalogram (EEG) and the monitoring of somatosensory evoked potentials. The use of neuromonitoring can guide the duration of the cooling period (e.g., isoelectric EEG) and can also provide evidence of an intraoperative stroke prior to skin closure,
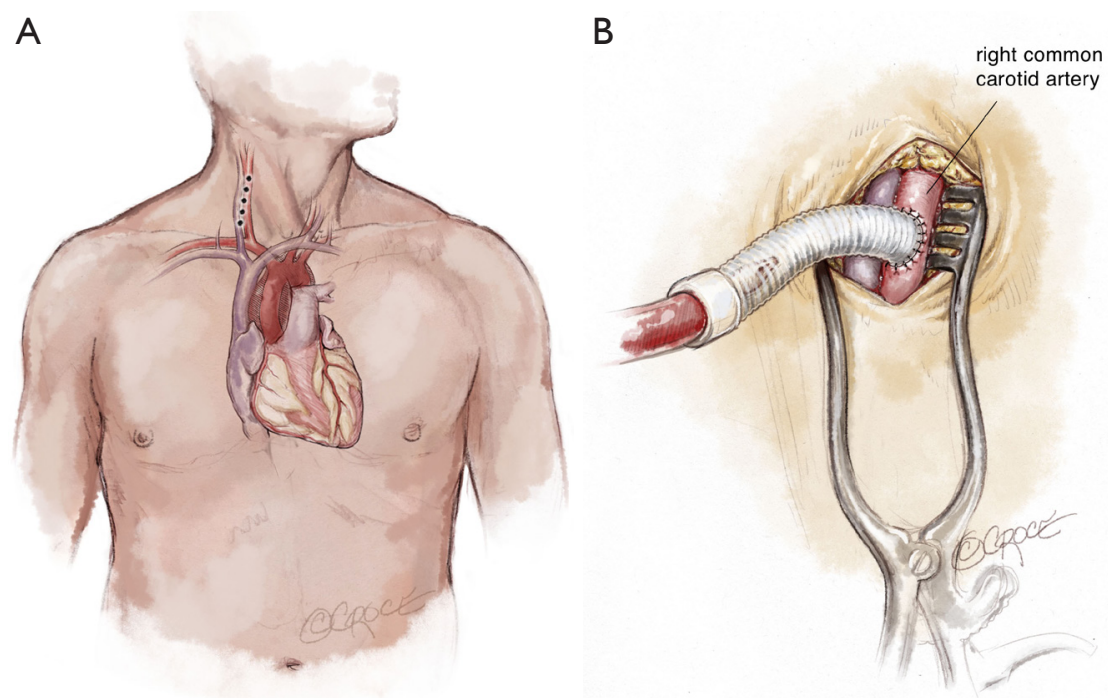

Figure 4 Common carotid artery cannulation. A $5 \mathrm{~cm}$ incision is made on the anterior border of the sternocleidomastoid, $2 \mathrm{~cm}$ above the clavicular head, and the carotid sheath is incised to expose the common carotid artery. An 8 mm Dacron graft is sewn end-to side on to the common carotid artery and cannulated for arterial inflow for cardiopulmonary bypass. 

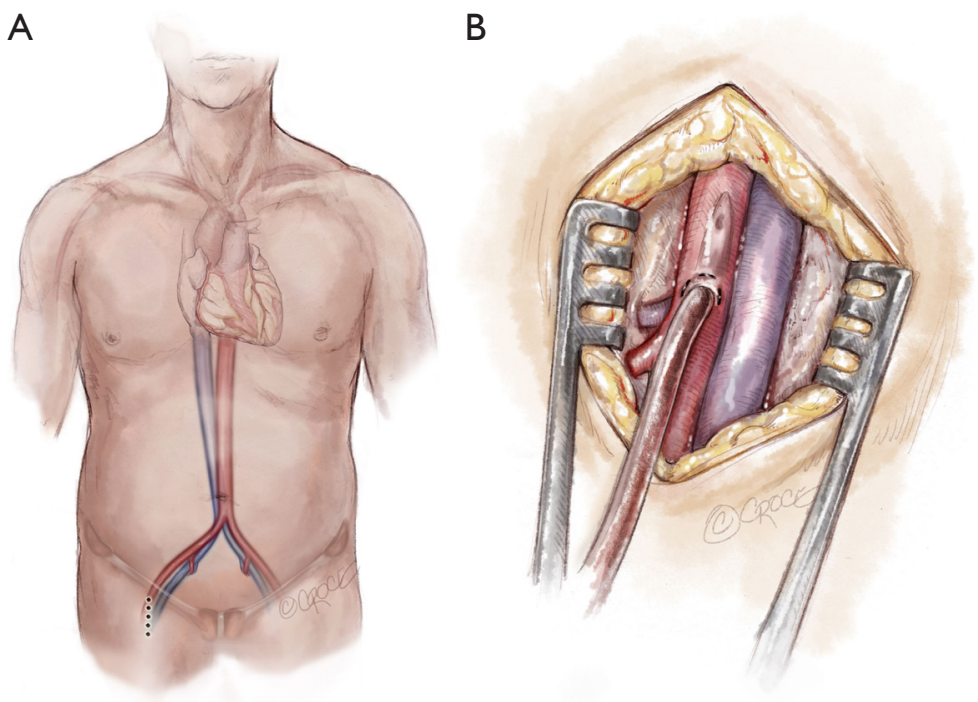

Figure 5 Femoral artery cannulation. A $5 \mathrm{~cm}$ vertical incision is made in the groin and the femoral artery is isolated and directly cannulated for arterial inflow for cardiopulmonary bypass.

which may prompt aggressive neurointerventions in the early postoperative period.

The simplest form of neuroprotection for surgical repair of ATAIMH is the use of deep hypothermic circulatory arrest alone (DHCA, nasopharyngeal temperature 14.1$\left.20.0^{\circ} \mathrm{C}\right)(10)$. Hypothermia has been shown to exponentially reduce cerebral metabolism, and a nasopharyngeal temperature of $20{ }^{\circ} \mathrm{C}$ has been associated with a $76 \%$ reduction of the cerebral metabolic rate (11). The strategy of DHCA can be used with any arterial cannulation site and provides a "safe" period of cerebral protection for 30 minutes of circulatory arrest. Longer durations of DHCA alone have been associated with a significant increase in adverse neurologic events $(12,13)$.

A second strategy that complements the use of hypothermia for neuroprotection during circulatory arrest is the use of adjunctive cerebral perfusion. The use of antegrade or retrograde cerebral perfusion (RCP) during the period of hypothermic circulatory arrest has significantly improved neurologic outcomes in patients undergoing arch reconstruction, especially for patients requiring extended periods of circulatory arrest.

RCP can be utilized with any arterial cannulation site. RCP is delivered by cannulating and snaring the superior vena cava, and infusing hypothermic blood at a temperature of $12-18{ }^{\circ} \mathrm{C}$ from the cardiopulmonary bypass circuit up the superior vena cava to perfuse the brain in a retrograde direction during the period of circulatory arrest. This can be accomplished in many different ways, including the insertion of a "bridge" between the arterial and venous limbs of the cardiopulmonary bypass circuit. At the time of circulatory arrest, clamps are placed distal to the bridge on the arterial side, proximal to the bridge on the venous side, and on the inferior vena cava venous line. Generally accepted flow rates are $300-500 \mathrm{~mL} / \mathrm{min}$ to maintain a superior vena cava pressure of $25-30 \mathrm{mmHg}$ (Figure 6). If performed properly, RCP will produce dark blood (suggesting cerebral oxygen extraction) flowing retrograde from the origins of the great vessels into the open aortic arch during circulatory arrest. RCP provides cerebral protection by: (I) flushing embolic material (gaseous and particulate) from the cerebral circulation; (II) maintaining cerebral hypothermia by bathing the brain in cold blood; (III) supporting cerebral metabolism by providing cerebral blood flow during the period of circulatory arrest. RCP is most commonly combined with the use of deep hypothermia and serves as a highly effective neuroprotection strategy for aortic arch reconstruction.

ACP refers to the delivery of blood at $12-18{ }^{\circ} \mathrm{C}$ to the brain during HCA in an antegrade direction, at a flow rate of $10 \mathrm{cc} / \mathrm{kg} / \mathrm{min}$, up the carotid arteries. This can be accomplished by several techniques using a variety of arterial cannulation sites. If the arterial cannulation site is the right axillary or innominate artery, unilateral ACP can be delivered by occluding the base of the innominate and left common carotid arteries during the period of 


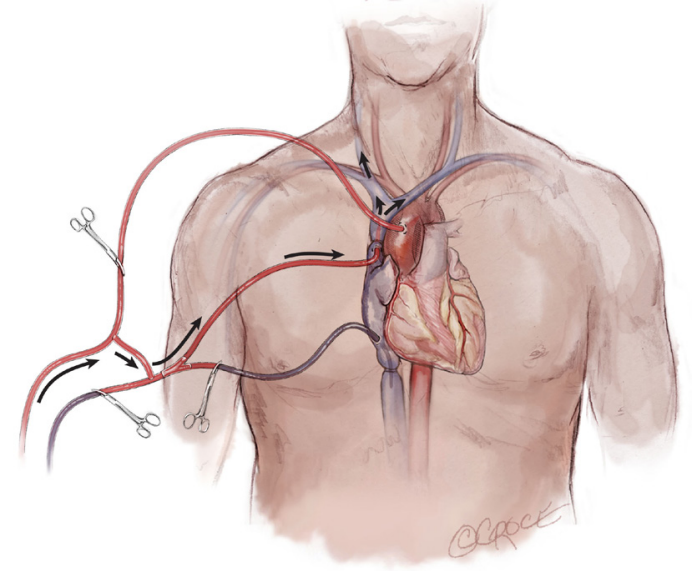

Figure 6 Retrograde cerebral perfusion. At the time of hypothermic circulatory arrest, clamps are placed distal to the bridge on the arterial side, proximal to the bridge on the venous side, and on the inferior vena cava venous limb. Generally accepted flow rates are $300-500 \mathrm{~mL} / \mathrm{min}$ to maintain a superior vena cava pressure of 25-30 $\mathrm{mmHg}$.

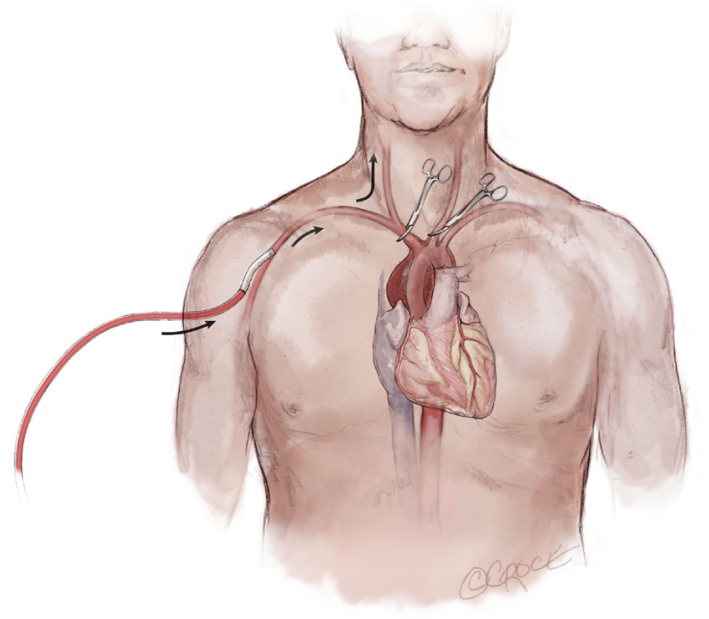

Figure 7 Antegrade cerebral perfusion. At the time of hypothermic circulatory arrest, the arterial inflow is lowered to $10 \mathrm{~mL} / \mathrm{kg} / \mathrm{min}$ and clamps are placed at the origins of the innominate and left common carotid arteries to initiate unilateral antegrade cerebral perfusion.

HCA (Figure 7). If the site of cannulation is the right or left carotid artery, unilateral ACP is initiated by occlusion of the common carotid artery proximal to the cannulation site. When the ascending aorta or femoral artery is the site of arterial cannulation, ACP can be initiated after the arch is opened during the period of circulatory arrest by the introduction of balloon tip perfusion catheters directly into the ostia of the innominate and/or left common carotid arteries, or by placing a cannula directly into the innominate artery and occluding it proximally (14). Unilateral ACP refers to antegrade flow delivered up one carotid artery (regardless of cannulation site), and bilateral ACP involves the insertion of a perfusion cannula into the ostium of the contralateral carotid after the arch has been opened. Neurologic outcomes associated with the use of unilateral $v s$. bilateral ACP have been shown to be equivalent (15).

It should also be recognized that the utilization of ACP changes the paradigm of circulatory arrest from the interruption of perfusion to all organs, to lower body circulatory arrest alone, as the brain, arms and the spinal cord (via collateral circulation) remain perfused. Therefore, the legs and the abdominal viscera are the sole ischemic organs during the circulatory arrest period with the use of ACP. The metabolic rate of the visceral organs and skeletal muscle is significantly less than the brain; thus, the visceral organs require a reduced degree of hypothermia for optimal protection and are more tolerant of ischemia. This is important to recognize because ACP is most commonly used in conjunction with moderate hypothermic circulatory arrest (MHCA, nasopharyngeal temperatures $20.1-28.0{ }^{\circ} \mathrm{C}$ ) as opposed to deep hypothermic circulatory arrest (10). MHCA/ACP has been shown to be an effective form of cerebral protection for acute ascending aortic syndromes, and is widely accepted as the optimal strategy for complex arch reconstruction which requires extended ( $>40$ minutes) circulatory arrest times $(16,17)$.

\section{Conclusions}

Proximal aortic replacement using hypothermic circulatory arrest continues to be the optimal treatment of ATAIMH. The illustrations and content of this manuscript should serve as a reference to provide several different options for arterial cannulation and neuroprotection. Patient anatomy and clinical status should be strongly considered when determining the circulation management strategy prior to incision. All of the described techniques have a role in the successful management of patients with ATAIMH, depending upon the clinical situation.

\section{Acknowledgments}

None. 


\section{Footnote}

Conflicts of Interest: The author has no conflicts of interest to declare.

\section{References}

1. Erbel R, Aboyans V, Boileau C, et al. 2014 ESC Guidelines on the diagnosis and treatment of aortic diseases. Eur Heart J 2014;35:2873-926.

2. Hiratzka LF, Bakris GL, Beckman JA, et al. 2010 ACCF/AHA/AATS/ACR/ASA/SCA/SCAI/SIR/STS/

SVM Guidelines for the Diagnosis and Management of Patients With Thoracic Aortic Disease. Circulation 2010;121:e266-369.

3. Evangelista A, Mukherjee D, Mehta RH, et al. Acute Intramural Hematoma of the Aorta. A Mystery in Evolution. Circulation 2005;111:1063-70.

4. Sandhu HK, Tanaka A, Charlton-Ouw KM, et al. Outcomes and management of type A intramural hematoma. Ann Cardiothorac Surg 2016;5:317-27.

5. Svensson LG, Blackstone EH, Rajeswaran J, et al. Does the Arterial Cannulation Site for Circulatory Arrest Influence Stroke Risk? Ann Thorac Surg 2004;78:1274-84; discussion 1274-84.

6. David TE, Armstrong S, Ivanov J, et al. Surgery for Acute Type A Aortic Dissection. Ann Thorac Surg 1999;67:1999-2001.

7. Nguyen B, Müller M, Kipfer B, et al. Different techniques of distal aortic repair in acute type A dissection: impact on late aortic morphology and reoperation. Eur J Cardiothorac Surg 1999;15:496-500; discussion 500-1.

8. Pugliese P, Pessotto R, Santini F, et al. Risk of late reoperations in patients with acute type A aortic dissection: impact of a more radical surgical approach. Eur J Cardiothorac Surg 1998;13:576-80; discussion 580-1.
9. Comas GM, Leshnower BG, Halkos ME, et al. Acute type A dissection: impact of antegrade cerebral perfusion under moderate hypothermia. Ann Thorac Surg 2013;96:2135-41.

10. Yan TD, Bannon PG, Bavaria J, et al. Consensus on hypothermia in aortic arch surgery. Ann Cardiothorac Surg 2013;2:163-8.

11. McCullough JN, Zhang N, Reich DL et al. Cerebral metabolic suppresion during hypothermic circulatory arrest in humans. Ann Thorac Surg 1999;67:1895-9.

12. Gega A, Rizzo JA, Johnson MH, et al. Straight Deep Hypothermic Arrest: Experience in 394 Patients Supports Its Effectiveness as a Sole Means of Brain Preservation. Ann Thorac Surg 2007;84:759-66.

13. Svensson LG, Crawford ES, Hess KR, et al. Deep hypothermia with circulatory arrest. Determinants of stroke and early mortality in 656 patients. J Thorac Cardiovasc Surg 1993;106:19-28.

14. Jassar AS, Vallabhajosyula P, Bavaria JE, et al. Direct innominate artery cannulation: An alternate technique for antegrade cerebral perfusion during aortic hemiarch reconstruction. J Thorac Cardiovasc Surg 2016;151:1073-8.

15. Zierer A, Risteski P, El-Sayed Ahmad A, et al. The impact of unilateral versus bilateral antegrade cerebral perfusion on surgical outcomes after aortic arch replacement: a propensity-matched analysis. J Thorac Cardiovasc Surg 2014;147:1212-7.

16. Leshnower BG, Kilgo PD, Chen EP. Total arch replacement using moderate hypothermic circulatory arrest and unilateral selective antegrade cerebral perfusion. J Thorac Cardiovasc Surg 2014;147:1488-92.

17. Leshnower BG, Myung RJ, Kilgo PD, et al. Moderate hypothermia and unilateral selective antegrade cerebral perfusion: a contemporary cerebral protection strategy for aortic arch surgery. Ann Thorac Surg 2010;90:547-54.
Cite this article as: Leshnower BG. Cannulation strategies, circulation management and neuroprotection for type A intramural hematoma: tips and tricks. Ann Cardiothorac Surg 2019;8(5):561-566. doi: 10.21037/acs.2019.08.08 